\title{
Diagnose und aktuelle Therapie von Schwindelsyndromen
}

Michael Strupp, Thomas Brandt

\begin{abstract}
Schwindel ist ein Anzeichen dafür, dass eine Störung im engeren oder weiteren Bereich des Gleichgewichtssystems vorliegt. Rund 30\% der Menschen kennen dieses Symptom als Krankheit - vor allem ältere Patienten. Die Ursachen für Schwindel sind vielfältig. Mit einer sorgfältigen und systematischen Anamnese und einer standardisierten körperlichen Untersuchung lassen sich die meisten Syndrome relativ einfach diagnostisch richtig einordnen und wirkungsvoll behandeln.
\end{abstract}

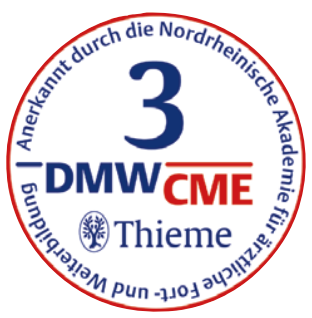

Erhebliche Unterversorgung I Eine aktuelle große epidemiologische Studie aus den USA zeigt, dass die jährliche Inzidenz von Schwindel bei über $11 \%$ liegt und die Mortalität der Betroffenen gegenüber Nicht-Betroffenen 1,7-fach höher ist [1]. Die Lebenszeitprävalenz beträgt mindestens 30\% [2]. Trotz dieser hohen klinischen Relevanz besteht unverändert eine erhebliche Unter-, aber auch Fehlversorgung [3]. Häufig werden zur Diagnose nicht nötige apparative Untersuchungen ohne gezielte Fragestellung durchgeführt und die eingesetzten Therapeutika sind entweder unwirksam oder wirken rein symptomatisch.

Fundierte Kenntnisse notwendig I Die korrekte Diagnose sollte rasch gestellt und eine wirksame Therapie eingeleitet werden, um so die Krank-

Tab. 1 Absolute und relative Häufigkeiten der verschiedenen Schwindelsyndrome in einer interdisziplinären Spezialambulanz für Schwindel ( $n=23915)$.

$\begin{array}{lll}\text { Diagnose } & \text { n } & \% \\ \begin{array}{l}\text { benigner peripherer paroxysmaler } \\ \text { Lagerungsschwindel }\end{array} & 3740 & 15,6 \\ \text { funktioneller Schwindel } & 3589 & 15,0 \\ \text { zentral-vestibulärer Schwindel } & 3182 & 13,3 \\ \text { vestibuläre Migräne } & 2829 & 11,8 \\ \text { Morbus Menière } & 2397 & 10,0 \\ \text { unilaterale Vestibulopathie } & 2130 & 8,9 \\ \text { bilaterale Vestibulopathie } & 1604 & 6,7 \\ \text { Vestibularisparoxysmie } & 816 & 3,4 \\ \text { psychogener Schwindel } & 614 & 2,6 \\ \text { Perilymphfistel / Bogengangsdehiszenz } & 146 & 0,6 \\ \text { unklare Schwindelsyndrome } & 883 & 3,7 \\ \text { andere* } & 1985 & 8,3 \\ * & \text { andere Schwindelsyndrome sind z. B. nicht-vestibulärer Schwindel bei } \\ \text { neurodegenerativen Erkrankungen oder nicht-vestibuläre Okulomotorikstö- } \\ \text { rungen bei Myasthenia gravis oder peripheren Augenmuskelparesen }\end{array}$

heitsdauer zu verkürzen und Funktionsfähigkeit sowie Lebensqualität der Patienten zu verbessern. Dafür sollten Ärzte verschiedener Fachrichtungen, insbesondere Hausärzte, Internisten, Neurologen und HNO-Ärzte über fundierte Kenntnisse und Fertigkeiten auf diesem - eigentlich ganz einfachen - Gebiet verfügen.

Formen des Schwindels I Schwindel ist ein Leitsymptom verschiedener Erkrankungen unterschiedlicher Ätiologie. Es lassen sich vier Formen unterscheiden:

1. Störungen des peripheren vestibulären Systems (Innenohr und/oder Gleichgewichtsnerv)

2. Störungen des zentralen vestibulären Systems (Hirnstamm oder Kleinhirn)

3. funktioneller Schwindel, wie der phobische Schwankschwindel

4. andere Ursachen wie Blutdruckregulationsstörungen oder unerwünschten Wirkungen von Medikamenten

Die vierte Gruppe wird meist überschätzt. - Tab. 1 zeigt die relative Häufigkeit der einzelnen Schwindelursachen in einer neurologischen Spezialambulanz.

\section{Diagnose}

Anamnese I Die meisten Schwindelsyndrome lassen sich nach sorgfältiger und systematischer Anamnese und körperlicher neurologischer, neuro-otologischer und neuro-ophthalmologischer Untersuchung diagnostisch richtig einordnen auch ohne viele apparative Zusatzuntersuchungen. Die vier wichtigen Unterscheidungskriterien der verschiedenen Schwindelsyndrome sind:

1. Dauer der Symptome

2. Art der Symptome

3. modulierende Faktoren (Auslösbarkeit/Verstärkung / Abschwächung)

4. mögliche Begleitsymptome (otogene, zentrale, migränetypische, vegetative u.a.) 


\section{Dauer der Symptome}

Sekunden bis Stunden I Schwindelattacke über:

- Sekunden bis wenige Minuten, z.B. benigner peripherer paroxysmaler Lageschwindel (BPPV $<1 \mathrm{~min}$ ), Vestibularisparoxysmie ( $<1 \mathrm{~min}$ ), Bogengangsdehiszenz (knöcherner Defekt des meist anterioren Bogengangs), paroxysmale Hirnstammattacken, orthostatische Dysregulation, transitorische ischämische Attacke (TIA)

- viele Minuten bis Stunden, z. B. Morbus Menière (20 min-12 h), vestibuläre Migräne (5 min$72 \mathrm{~h}$ ), episodische Ataxien.

Die Schwindelattacken beruhen meist auf einer pathologischen einseitigen Hemmung oder Erregung des bilateral angelegten peripheren oder zentralen vestibulären Systems.

Tage bis Wochen I Akut einsetzende über Tage bis wenige Wochen anhaltende Symptome (z.B. akute einseitige Vestibulopathie, Hirnstammoder Kleinhirninfarkt). Die Symptome werden durch eine meist einseitige periphere oder zentrale Läsion verursacht. Pathophysiologisch lassen sich die Symptome durch eine vestibuläre Tonusimbalance erklären, die über Tage bis wenige Wochen zentral kompensiert wird.

Monate bis Jahre I Über Monate bis Jahre anhaltende Symptome, z.B. bilaterale oder persistierende unilaterale Vestibulopathie, funktioneller Schwindel, neurodegenerative Erkrankungen, die das cerebelläre System (cerebelläre Ataxien, Downbeat-Nystagmussyndrom) oder extrapyramindale System (Multisystematrophie, Parkinsonsyndrome) betreffen. Diese Symptome werden bei einer peripheren vestibulären Läsion durch das sensorische Defizit verursacht, bei funktionellem Schwindel durch die Selbstbeobachtung der Balance und bei zentralen Erkrankungen durch Störungen der Koordination.

\section{Art der Schwindelsymptome}

- Drehschwindel I Wie Karussellfahren (z.B. BPPV, akute einseitige Vestibulopathie). Typischerweise entsteht isolierter Drehschwindel wie bei einem BPPV durch eine Störung der Funktion der Bogengänge, da diese Drehbeschleunigungen detektieren, oder durch eine Störung des vestibulären Nervs oder Kerngebietes.

- Schwankschwindel | Wie Bootfahren (z.B. bilaterale Vestibulopathie, posttraumatischer Otolithenschwindel oder funktioneller Schwindel). Ein sensorisches Defizit manifestiert sich als bewegungsabhängiger Schwankschwindel. Isolierter Schwankschwindel findet sich auch beim posttraumatischen Otolithenschwindel, aber auch nach erfolgreichen Befreiungsmanövern beim BPPV durch die partielle Reposition der Otokonien auf den Utrikulus [4].

- Benommenheitsschwindel | Z.B. funktioneller Schwindel oder unerwünschte Medikamentenwirkung.

\section{Modulierende Faktoren}

„Ruheschwindel“ I Schwindel bereits in Ruhe vorhanden oder spontan auftretend (z.B. akute einseitige Vestibulopathie, Hirnstamm- oder Kleinhirninfarkt, Morbus Menière, Vestibularisparoxysmie). Die Symptome werden durch die vestibuläre Tonusimbalance verursacht und verstärken sich bei Bewegung.

Schwindel beim Stehen oder Gehen | Z. B. bilaterale oder persistierende einseitige Vestibulopathie, funktioneller Schwindel. Die Beschwerden beruhen bei den Vestibulopathien auf dem sensorischen Defizit, wobei die Patienten typischerweise keine Symptome in Ruhe im Liegen oder Sitzen haben. Dies ist eine wichtige Frage zur Differenzialdiagnose.

Auslösung des Schwindels | Der Schwindel lässt sich auslösen durch:

- Kopflageänderung relativ zur Schwerkraft (z.B. BPPV oder zentraler Lagenystagmus)

- horizontale Kopfdrehungen (z.B. Vestibularisparoxysmie, „rotational vertebral artery occlusion syndrome“)

- Husten, Pressen, Niesen oder Heben schwerer Lasten (z. B. Bogengangsdehiszenz, Perilymphfistel)

- bestimmte soziale Situationen wie Menschenmengen, Kaufhaus (z.B. phobischer Schwankschwindel)

Besserung der Symptome I Typisch für funktionellen Schwindel ist, wenn sich Symptome nach leichtem Alkoholgenuss, beim Sport und bei Ablenkung bessern und kaum oder keine Symptome morgens nach dem Aufwachen bestehen [5].

\section{Mögliche Begleitsymptome}

„Otogene“ Symptome | Z.B. attackenartig verstärkter Tinnitus oder Hypakusis, die für einen M. Menière sprechen, aber auch seltener bei Hirnstammischämien auftreten können. Eine Autophonie, d.h. verstärktes Hören körpereigener Geräusche, findet sich beim Bogengangsdehiszenzsyndrom.

Potenzielle Hirnstammsymptome I Wie Doppelbilder, Gefühlsstörungen im Gesicht oder an den Extremitäten, Schluck-, Sprechstörungen, Lähmungen oder Feinmotorikstörungen (diese Symptome deuten auf eine zentrale, meist Hirnstammläsion hin). 


\section{Art der Untersuchung Inspektion}

Körper- und Kopfhaltung

Stellung der Augenlider

\section{Augenposition /-motilität}

Stellung der Augen beim Geradeausblick

Abdeck (Cover)-Test

Untersuchung der Augen in den 8 Endpositionen (bin- und monokulär), d. h. rechts, links, oben, unten und in den 4 Diagonalen

\section{Blickhaltefunktion}

Blick nach etwa $10^{\circ}-40^{\circ}$ horizontal bzw. $10^{\circ}-20^{\circ}$ vertikal und zurück nach $0^{\circ}$

\section{langsame Blickfolge}

horizontal und vertikal

\section{Sakkaden}

horizontal und vertikal beim Umherblicken und gezielter Aufforderung

\section{optokinetischer Nystagmus}

horizontal und vertikal mit OKN-Trommel, Streifenband oder „Optodrum“

\section{periphere vestibuläre Funktion}

klinische Testung des vestibulo-okulären-Reflexes

\section{Fixationssuppression des vestibulo-okulären-Reflexes}

Fixation eines Blickziels während Drehung des Kopfes und des Blickziels mit derselben Winkelgeschwindigkeit

\section{Untersuchung unter der M-Brille}

Blick geradeaus

Kopfschütteltest („Head-shaking test“)

Lagerungsmanöver

Tab. 2 Klinischer Untersuchungsgang des okulomotorischen und vestibulären Systems. Wichtig ist ein standardisiertes Vorgehen, das meist eine Differenzierung zwischen zentralen und peripheren okulomotorischen und vestibulären Störungen ermöglicht. $\mathrm{BBPV}=$ benigner peripherer paroxysmaler Lagerungsschwindel
Frage nach ...

Neigung oder Drehung des Kopfes / Körpers

Ptose

primärer Fehlstellung, Spontan-, Fixationsnystagmus

horizontaler oder vertikaler Fehlstellung, latentem Nystagmus

Bewegungsausmaß, Endstellnystagmus

Blickrichtungsnystagmus: horizontal und vertikal, ReboundNystagmus

glatt oder sakkadiert

Latenz, Geschwindigkeit, Zielgenauigkeit und konjugierten Bewegungen

Auslösbarkeit, Schlagrichtung und Phase (Umkehrung?)

ein- oder beidseitiger peripherer vestibulärer Läsion

fehlender Unterdrückung des vestibulo-okulären-Reflexes als Zeichen einer zerebellären Störung

peripherem vestibulärem Spontannystagmus, typischerweise durch Fixation unterdrückt, oder zentralem Fixationsnystagmus, der durch Fixaton nicht unterdrückt wird

Kopfschüttelnystagmus bei einseitiger peripherer vestibulärer Läsion zum nicht betroffenen Labyrinth; selten sog. cross-coupling als zentrales Zeichen

Lagerungsnystagmus beim BPPV, DD zentraler Lagenystagmus
Kopfschmerzen | Episodischer Kopfschmerz und/oder Licht- oder Lärmempfindlichkeit, visuelle Aura zusammen mit den Schwindelbeschwerden oder bekannte Migräne deuten auf eine vestibuläre Migräne hin. Kopfschmerz kann natürlich aber auch bei einer Hirnstammischämie oder Blutungen in die hintere Schädelgrube auftreten.

Scheinbewegungen der Umgebung I Diese sogenannten Oszillopsien finden sich spontan bei Patienten mit Nystagmus (Ausnahme: kongenitaler/infantiler Nystagmus) oder beim Gehen und Kopfbewegungen bei Patienten mit bilateraler Vestibulopathie aufgrund des vestibulo-okulären-Reflexes(VOR)-Defizits.
Übelkeit, Erbrechen I Hierbei handelt es sich um unspezifische Begleitsymptome, die sowohl bei akuten peripheren als auch zentralen vestibulären Störungen, aber selten auch bei funktionellen Störungen vorkommen können.

\section{Körperliche Untersuchung}

Klinische Tests | Das vestibuläre System lässt sich mittels fünf klinischer Tests gut untersuchen $(\triangleright$ Tab. 2):

1. Kopfimpulstest nach Halmagyi-Curthoys | Mit der Frage nach einem ein- oder beidseitigen 


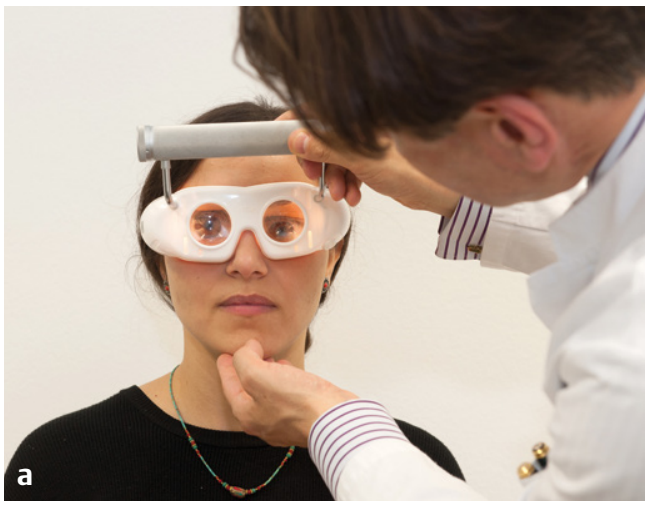

Funktionsdefizit des VOR [6] oder Video-Kopfimpulstest (Video-HIT; s. u.), der der klinischen Untersuchung deutlich überlegen ist [7]. Zur Prüfung des horizontalen VOR hält man den Kopf des Patienten zwischen beide Hände, bittet diesen, ein Ziel zu fixieren und führt schnelle horizontale Kopfbewegungen nach rechts und links durch. Beim Gesunden führen diese Kopfrotationen zu raschen entgegengesetzten kompensatorischen Augenbewegungen, die die gleiche Geschwindigkeit haben, wie die Kopfbewegung, so dass das Bild auf der Retina stabil bleibt. Bei einem Labyrinthausfall bewegen sich die Augen bei Kopfrotationen zur betroffenen Seite mit dem Kopf mit. Der Patient muss deshalb eine Sakkade machen, um das Ziel wieder fixieren zu können. Diese Refixationssakkade ist das klinische Zeichen für ein Defizit des VOR. Auf diese Weise können ein- und beidseitige Störungen des VOR diagnostiziert werden.

2. Untersuchung auf einen peripheren vestibulären Spontannystagmus mittels Frenzelbrille/MBrille | Dies ermöglicht eine Differenzierung zwischen einem zentralen Fixationsnystagmus, der sich typischerweise nicht durch Fixation unterdrücken lässt und einen peripheren vestibulären Spontannystagmus wie bei einer akuten einseitigen Vestibulopathie ( $\checkmark$ Abb. 1 ). Die M-Brille ist ein neues leichtes ( $8 \mathrm{~g})$ Untersuchungsinstrument mit vergrößernden Fresnel-Linsen (+20 dpt.), das mittels der elastischen Bügel an der Nase selbständig sitzt [8]. Die Linsen reduzieren die visuelle Fixation, die typischerweise einen peripheren vestibulären Spontannystagmus unterdrückt („visuelle Fixationssuppression“). Ferner erleichtern sie die Beobachtung der Augenbewegungen des Patienten auch ohne innere Beleuchtung. Achten sollte man bei der Untersuchung mittels der M-Brille auf

- einen peripheren vestibulären Spontannystagmus,

- einen Kopfschüttelnystagmus (dazu bittet man den Patienten, seinen Kopf 20 mal schnell nach rechts und links zu drehen, anschließend Beobachtung der Augenbewegungen)

- einen Lagerungs- oder seltenen zentralen Lagenystagmus

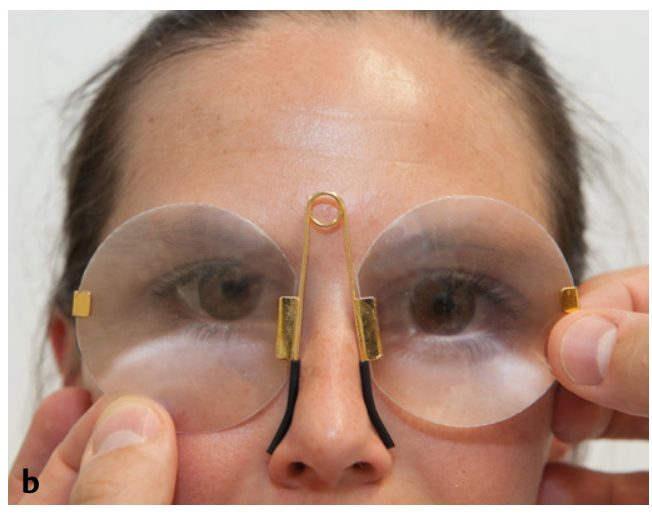

Abb.1 Klinische Untersuchung auf Nystagmus mittels

(a) Frenzelbrille oder

(b) M-Brille.
Der Spontannystagmus zeigt eine Tonusimbalance des VOR an, wie z.B. bei einer akuten einseitigen Vestibulopathie. Der Kopfschüttelnystagmus zeigt eine latente Asymmetrie des sog. velocity storage an. Sie kann sowohl auf peripheren als auch selten auf zentralen vestibulären Funktionsstörungen beruhen.

3. Lagerungsmanöver | Mit der Frage nach einem BPPV oder einem zentralen Lagerungs-/ Lagenystagmus. Wichtigstes Unterscheidungskriterium zwischen beiden ist die Richtung des ausgelösten Nystagmus:

- Bei einem BPPV entspricht die Richtung des Nystagmus der Ebene des betroffenen Bogengangs: vertikal-torsionell beim posterioren $\mathrm{Ka}$ nal, linear horizontal beim horizontalen Bogengang

- bei einem zentralen Lagerungsnystagmus findet sich in unterschiedlichen Kopfpositionen eine jeweils sehr ähnliche Nystagmusrichtung, meistens in Form eines Downbeat-Nystagmus.

4. Untersuchung auf das Vorliegen der möglichen 4 Komponenten einer "ocular tilt reaction“

1. Auslenkung der subjektiven visuellen Vertikalen (SVV) mittels des "Eimertests" [9] ( Abb. 2). Dies ist ein empfindlicher Test für eine akute einseitige vestibuläre Läsion, der aber nicht zwischen peripheren und zentralen Störungen differenziert

2. Kopfverkippung (diese findet sich aber auch bei einer Trochlearisparese und zwar mit Beugung zur gesunden Seite)

3. vertikale Divergenz der Augen (sog. „skew deviation“) mit dem alternierenden Abdecktest. Eine vertikale Divergenz findet sich praktisch nur bei zentralen Läsionen, aber nicht alle zentralen Läsionen gehen mit einer vertikalen Divergenz einher. Die Häufigkeit bei akuten Hirnstamminfarkten liegt bei $30 \%[10,11]$

4. Augenverrollung mit dem Scanning-Laser Ophthalmoskop (dies ist für die Routine weniger wichtig)

5. Untersuchung des Stand- und Gehvermögens | Mit offenen und geschlossenen Augen und verschiedenen Schwierigkeitsgraden (Füße nebenei- 
Abb. 2 Bestimmung der subjektiven visuellen Vertikalen (SVV) mit dem „Vertikalentest mit SVV-Eimer“ [9]. (a) Der Patient schaut in den Eimer auf eine an der Unterseite angebrachte Linie, die er senkrecht einstellen muss. (b) Der Untersucher liest dann die Ablenkung von der tatsächlichen Vertikalen („Lot“) ab. Es sollten 10 Einzelmessungen stattfinden. Der Referenzbereich $(x \pm 2 S D)$ liegt bei $0 \pm 2,5^{\circ}$.

Tab. 3 Topographische Anatomie okulomotorischer Störungen des Hirnstamms und Kleinhirns.

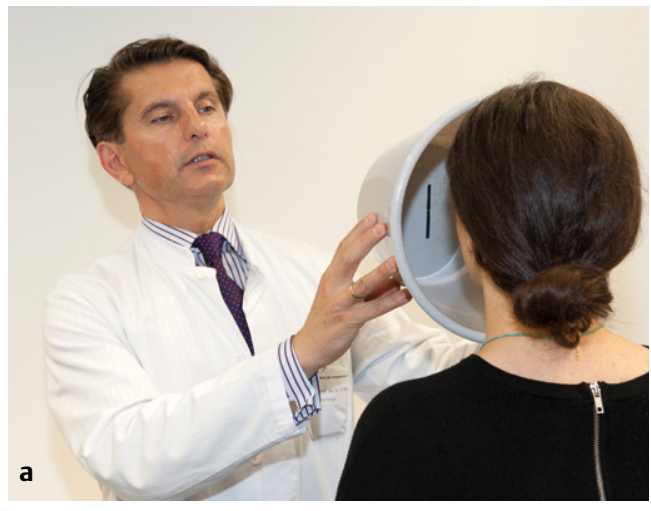

nander, Tandem-Romberg, Stehen auf einem Bein), mit der Frage nach sensorischen Defiziten, und zwar des vestibulären oder somatosensorischen Systems.

\section{Untersuchung des Hörvermögens und der Okulomotorik}

Auf Hörminderung prüfen | Klinisch kann dies durch Reibegeräusche und mittels Stimmgabel im Seitenvergleich erfolgen. Bei Verdacht auf eine Störung ist insbesondere für die Diagnose eines $M$. Menière eine audiometrische Testung erforderlich. Bei letzterem wird eine Hörminderung

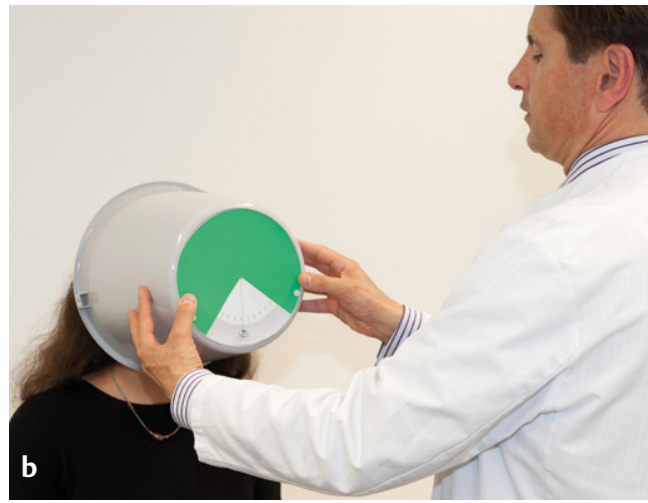

unter $2000 \mathrm{~Hz}$ von mindestens $30 \mathrm{~dB}$ für die Diagnosestellung gefordert [12].

Akut peripher oder zentral? I Schon allein durch die Kombination von Anamnese und körperlichen Befunden lässt sich in den meisten Fällen die richtige Diagnose stellen, zumal die diagnostischen Kriterien auf diesen Informationen beruhen. Eine Unterscheidung zwischen akutem peripheren und zentralen Schwindel ist meist mittels „fünf klinischer Zeichen“ mit einer Sensitivität und Spezifität von über $90 \%$ möglich:

1. vertikale Divergenz („skew deviation“)

2. zentraler Fixationsnystagmus (versus peripherem vestibulärem Spontannystagmus)

\section{klinischer Befund: Okulomotorikstörungen und Nystagmus \\ isolierte vertikale Sakkadenparese \\ isolierte horizontale Sakkadenparese \\ isolierte einseitige horizontale Sakkadenparese \\ hypermetrische Sakkaden \\ isolierter vertikaler Blickrichtungsnystagmus, d. h. nach oben und unten}

isolierter Blickrichtungsnystagmus nach rechts und links

internukleäre Ophthalmoplegie

Downbeat-Nystagmus

Upbeat-Nystagmus

Konvergenzretraktionssnystagmus

\section{wahrscheinlicher Ort der Schädigung im Hirnstamm und Kleinhirn}

Mesencephalon (rostraler interstitieller Nucleus des medialen Längsbündels [riMLF])

Pons (paramediane pontine Formatio reticularis [PPRF])

Sakkadenparese jeweils ipsilateral zur Läsion

cerebellär

Mesencephalon (interstitieller Nucleus Cajal [INC], d. h. des neuronalen Integrators vertikaler und torsioneller Augenbewegungen)

Ponto-medullär / zerebellär (Nucleus präpositus hypoglossi, Vestibulariskerne, Vestibulozerebellum, d. h. des neuronalen Integrators horizontaler Augenbewegungen)

MLF-Läsion ipsilateral auf der Seite der Adduktionshemmung meist Zerebellum mit beidseitiger Störung des Flocculus Medulla oblongata oder Mesencephalon Mesencephalon (Commissura posterior)

Tab. 4 Relevante zerebelläre Strukturen und typische Okulomotorikstörungen und Nystagmusformen.

\section{Ort der Schädigung}

Flocculus/Paraflocculus

Nodulus/Uvula

Vermis und Nucleus fastigii

\section{typische Befunde}

sakkadierte Blickfolge, Downbeat-Nystagmus, Rebound-Nystagmus, Störung der visuellen Fixationssuppression des VOR zentraler Lagenystagmus, periodisch alternierender Nystagmus hypometrische / hypermetrische Sakkaden 


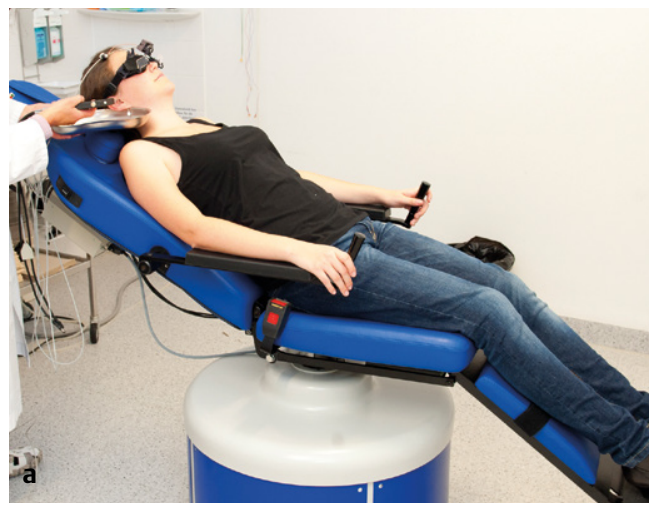

3. sakkadierte Blickfolge

4. Blickrichtungsnystagmus entgegen der Richtung des Spontannystagmus

5. normaler Kopfimpulstest bei akutem Schwindel und Nystagmus [11]

Okulomotorik | Die Untersuchung des okulomotorischen Systems ist zusammen mit der topographisch-anatomischen Diagnose in $>$ Tab. 3 zusammengefasst. Diese ist auch wichtig für die Differenzierung akuter peripherer von akuten zentralen Syndromen und sollte auch eine Untersuchung auf die verschiedenen Nystagmusformen umfassen und ist damit obligat [13].

\section{Apparative Untersuchungen des vestibulären Systems}

Übersicht | Die wichtigsten apparativen Zusatzuntersuchungen beim Leitsymptom Schwindel sind:

- Videookulographie (VOG) ( Abb. 3), inklusive kalorischer Prüfung und Kopfimpulstest mit quantitativer Messung des VOR

- vestibulär-evozierte myogene Potenziale zur Untersuchung der Otolithenfunktion

- Posturographie

Videookulographie | Diese dient der Registrierung von Augenbewegungen und Messung der Funktion des VOR. Die Videookulographie ist eine nicht invasive Methode, die in den letzten Jahren so weit entwickelt wurde, dass sie als valide und reliable Methode zur Registrierung von Augenbewegungen eingesetzt wird. Mittels einer oder zweier Videokameras (d.h. mon- oder binokulärer Registrierung), die in eine kopfgebundene Maske integriert sind, werden die Augen gefilmt. So lässt sich z.B. die Intensität eines Spontannystagmus messen, eine kalorische Testung durchführen ( $\bullet$ Abb. 4a) und in Kombination mit dem Kopfimpulstest die Funktion des VOR im Hochfrequenzbereich quantifizieren $(\checkmark$ Abb. 4b). Letzteres ist der Testung mit dem klinischen Kopfimpulstest deutlich überlegen [7].

Spezifische Rolle der Bildgebung bei vestibulären Erkrankungen I Die hochauflösende CT-Bildge-

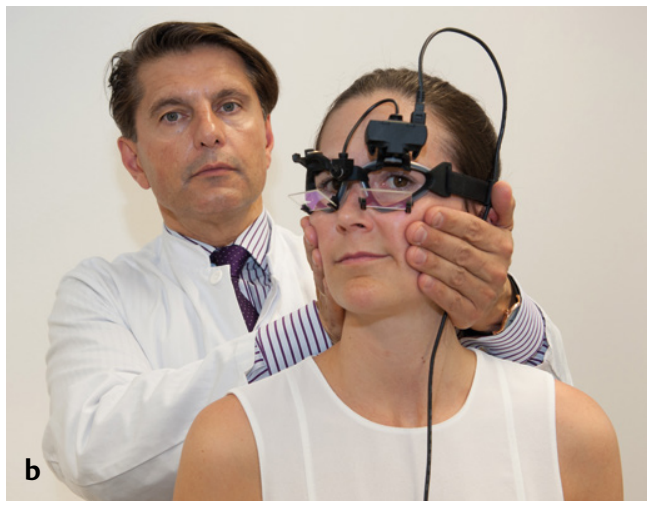

bung ist vor allem bei folgenden vestibulären Erkrankungen von Bedeutung:

- Bogengangsdehiszenzsyndrom (fehlende knöcherne Abdeckung des meist vorderen Bogengangs)

- Felsenbeinfrakturen

- hereditäre Labyrinthfehlbildungen

Kernspintomografie | Die hochauflösende Kernspintomografie ist wichtig zur Diagnose:

- zentraler Läsionen wie bei einer Pseudoneurits vestibularis (Infarkt oder MS-Plaque im Eintrittsbereich des N. vestibularis oder Vestibulariskerngebiet) oder Kleinhirninfarkten

- eines Vestibularisschwannoms, Gefäßnervkontaktes bei der Vestibularisparoxymie, Labyrinthfehlbildung und Endolymphhydrops bei Morbus Menière [14]

\section{Häufige Schwindelsyndrome und ihre spezifische Therapie}

Peripher vestibulärer Schwindel | Funktionell lassen sich drei Formen peripherer vestibulärer Störungen mit typischen Symptomen und klinischen Zeichen differenzieren:

1. der chronische beidseitige Ausfall des Nervus vestibularis und / oder der Vestibularorgane

2. die akute einseitige Vestibulopathie

3. paroxysmale pathologische Erregung oder Hemmung des Nervus vestibularis oder der Vestibularorgane

Im Folgenden werden Anamnese, Befund und aktuelle Therapie der fünf häufigsten peripher vestibulären Schwindelformen dargestellt.

\section{Benigner peripherer paroxysmaler Lageschwindel}

Häufigste Form I Der BBPV ist die häufigste Schwindelform, vor allem im höheren Alter ( $\triangleright$ Tab. 1). Die Lebenszeitprävalenz liegt bei mindestens 5\%. Der BPPV des posterioren Bogengangs ist charakterisiert durch kurze Drehschwindelattacken mit gleichzeitigem zum unten liegenden Ohr rotierendem und zur Stirn schla-
Abb. 3 Videookulographie (VOG). Mittels VOG lassen sich Augenbewegungen registrieren, um z. B. die Intensität eines Spontannystagmus zu messen. Um ein Defizit des vestibulookulären-Reflexes im niedrigen Frequenzbereich festzustellen, erfolgt die kalorische Prüfung mit kühlem und warmem Wasser (a). Um ein Defizit im hohen Frequenzbereich festzustellen, wird der Kopfimpulstest mit der Videookulographie kombiniert (b) mit synchroner Messung der Kopf- und Augenwinkelgeschwindigkeit. Der Video-HIT ist der klinischen Testung überlegen [7]. 
gendem vertikalem Lagerungsnystagmus, ausgelöst durch Kopfreklination oder Kopf- bzw. Körperseitlagerung zum betroffenen Ohr. Drehschwindel und Nystagmus treten nach der Lagerung mit einer kurzen Latenz von Sekunden in Form eines Crescendo-Decrescendo-Verlaufs von 30-60 Sekunden Dauer auf. Der Nystagmus entspricht einer sog. ampullofugalen Erregung des betroffenen hinteren vertikalen Bogengangs des unten liegenden Ohrs.

Fast immer idiopathisch | Über 90\% aller Fälle sind idiopathisch, während die symptomatischen Fälle am häufigsten auf ein Schädeltrauma, Neuritis vestibularis und Morbus Menière zurückgeführt werden. Der BPPV tritt auch auffällig häufig bei längerer Bettruhe bedingt durch andere Erkrankungen oder nach Operationen auf. 10\% der spontanen Fälle und 20\% der traumatischen Fälle zeigen einen beidseitigen, meist asymmetrisch betonten BPPV. Der seltenere BPPV des horizontalen Bogengangs wird durch Kopfdrehung im Liegen ausgelöst und ist durch einen linear horizontalen Nystagmus charakterisiert.

Benigne wird der BPPV genannt, weil er meist innerhalb von Wochen oder Monaten spontan abklingt.

In einigen Fällen kann er jedoch über Jahre anhalten. Unbehandelt persistiert der BPPV bei etwa $30 \%$ der Patienten über Wochen.

Canalolithiasishypothese I Diese kann alle Symptome des Lagerungsnystagmus erklären [15]. Anstelle fest auf der Cupula haftender Teilchen werden bei der Canalolithiasis frei im Bogengang bewegliche, aus vielen Otokonien zusammengesetzte und das Lumen des Bogengangs annähernd ausfüllende Konglomerate als Ursache des Lagerungsschwindels angenommen.

Therapie mit Befreiungsmanövern für den posterioren Bogengang I Durch rasche Kopflagerungen kann das Agglomerat aus dem Bogengang herausgespült werden und verursacht dann keinen Lageschwindel mehr [16]. Therapie der Wahl sind entweder das Sémont- oder Epley-Manöver [17]. Bei letzterem wird der liegende Patient in Kopfhängelage gedreht. Die meisten Patienten können diese Manöver nach kurzer Anleitung selbständig durchführen.

Beide Manöver sind gleichermaßen wirksam und die Heilungsrate liegt bei über $95 \%$ innerhalb weniger Tage.

Das jährliche Rezidivrisiko des BPPV liegt bei etwa $15-30 \%$ und im Verlauf kommt es bei ca. $50 \%$ der Patienten zu einem Rezidiv, das auf die gleiche Art erfolgreich behandelt werden kann.
Befreiungsmanöver nach Sémont | Am Beispiel einer Canalolithiasis des rechten posterioren Bogengangs:

1. In sitzender Ausgangsposition wird der Kopf um 45 Grad zum nicht betroffenen („gesunden“) linken Ohr gedreht.

2. Der Patient wird nach nach rechts gelagert, d. h. zum betroffenen Ohr unter Beibehaltung der Kopfrotation: Dies löst eine Bewegung der Teilchen im posterioren Bogengang entsprechend der Schwerkraft aus und führt zu einem vertikalen und rotierenden, erschöpflichen Nystagmus zum unten liegenden Ohr. Diese Position sollte der Patient ca. 1 Minute einnehmen.

3. Der Patient wird unter Beibehaltung der Kopfdrehung im raschen Schwung zum nicht betroffenen Ohr geworfen („großer Wurf“), wobei nun die Nase nach unten zeigt. Jetzt bewegen sich die Teilchen zum Ausgang des posterioren Bogengangs. Auch diese Position soll mindestens 1 Minute beibehalten werden.

4. Der Patient richtet sich langsam auf. Die Teilchen gelangen in den Utrikulusraum, wo sie keinen Drehschwindel mehr auslösen können. Diese Sequenz (1-4) sollte jeweils dreimal morgens, mittags und abends durchgeführt werden. In der Regel sind die Patienten so nach drei Tagen beschwerdefrei [16].

BPPV des horizontalen Bogengangs (hc-BPPV) I Die seltenere hc-BPPV-Variante (etwa 10\%) ist durch einen linearen horizontalen Nystagmus bei den Lagerungsmanövern gekennzeichnet, der bei einer Canalolithiasis zum unten liegenden $\mathrm{Ohr}$ schlägt (höhere Intensität auf der betroffenen Seite), bei einer Cupulolithiasis zum oben liegenden Ohr (höhere Intensität auf der nicht-betroffenen Seite). Für die Behandlung der Canalolithiasis des horizontalen Bogengangs sind diverse Behandlungsverfahren beschrieben, von denen die folgenden am häufigsten angewandt werden: - stufenweise $90^{\circ}$-Rotationen um die Körperlängsachse zum nicht-betroffenen $\mathrm{Ohr}$

- für $12 \mathrm{~h}$ auf dem nicht-betroffenen Ohr liegen - die Kombination von beiden [18]

Bei Vorliegen einer Kupololithiasis eines horizontalen Bogengangs, d.h. an der Cupula haftenden Otokonien, sollte diese erst in eine Canalolithiasis umgewandelt werden. Dies gelingt durch Schütteln des um 90 Grad nach vorne gebeugten und damit in die Horizontalebene gebrachten Kopfs [19]. Anschließend erfolgt dann eines der o.g. Befreiungsmanöver für eine Kanalolithiasis des horizontalen Bogengangs.

\section{Morbus Menière}

Diagnostische Kriterien | Diese sind [12]:

- zwei oder mehr Schwindelattacken von 20 min-12 h Dauer 
- nachgewiesene Hörminderung $(<2000 \mathrm{~Hz}$, mindestens $-30 \mathrm{~dB}$ ), assoziiert mit Schwindelattacke

- fluktuierender Tinnitus oder Ohrdruck im betroffenen $\mathrm{Ohr}$

- keine Hinweise für andere Ursache

Ätiologie und Pathophysiologie | Diese sind trotz vieler Untersuchungen bislang nicht sicher geklärt. Der pathognomonische histopathologische Befund ist ein Endolymphhydrops, der sich auch bildgebend mittels hochauflösender MRT des Felsenbeins nach transtympanaler oder intravenöser Injektion von Gadolinium gut darstellen lässt. Die Attacken entstehen wahrscheinlich durch eine Öffnung drucksensitiver Kationenkanäle und/oder die Ruptur der Endolymphmembran mit einer Erhöhung der Kaliumkonzentration im Perilymphraum, was zunächst zu einer Erregung, dann zu einer Depolarisation der Axone führt.

Verlauf I Die Erkrankung beginnt einseitig mit sehr unregelmäßiger, zunächst zunehmender, dann wieder abfallender Attackenfrequenz. Je länger man Patienten mit Morbus Menière verfolgt, desto häufiger sieht man bilaterale Erkrankungen in bis zu 50\% nach 20 Jahren. Der Verlauf ist bei vielen Patienten „benigne“ mit einer Abnahme der Attackenfrequenz innerhalb der ersten 5-10 Jahre, allerdings auch gleichzeitigem Auftreten von meist progredienten vestibulokochleären Defiziten.

Therapie | Ziel der prophylaktischen Behandlung ist es, den Endolymphhydrops zu vermindern. Trotz der hohen Prävalenz des Morbus Menière und vieler klinischer Studien gibt es bislang keine als sicher wirksam nachgewiesene, die Funktion nicht beeinträchtigende Therapie. Das Spektrum der Empfehlungen reicht von salzfreier Kost, über Diuretika, transtympanale Gentamycingabe (20$40 \mathrm{mg}$ in mehrwöchigem Abstand bis zur Symptombesserung) oder Betahistin bis zu operativen Verfahren. Positive Effekte auf die Attackenfrequenz wurden publiziert für die transtympanale Instillation von Gentamycin. Nachteile/Einschränkungen der Behandlung mit Gentamycin sind, dass sich nicht in allen Fällen das betroffene Ohr identifizieren lässt, es nach der Behandlung in etwa $20 \%$ zu einer Hörstörung kommt sowie das o.g. bilaterale Auftreten der Erkrankungen.

Betahistin-Therapie | Eine Therapie mit Betahistin in unterschiedlicher Dosierung wird ebenfalls diskutiert. Die BEMED-Studie zeigte jedoch weder einen Effekt von $2 \times 24 \mathrm{mg} / \mathrm{d}$ noch von $3 \times 48 \mathrm{mg} / \mathrm{d}$ [20]. Dennoch empfehlen die Autoren aufgrund eigener Erfahrungen („Expertenempfehlung“, niedrigstes Evidenzniveau!) noch höhere Dosierungen von mindestens $3 \times 96 \mathrm{mg}$ pro Tag über mind. 12 Monate. Bei manchen Patienten waren Tagesdosen von $480 \mathrm{mg} / \mathrm{d}$ notwendig und wirksam [21]. Als Wirkmechanismus wird eine Erhöhung des Blut- flusses im Innenohr angenommen [22] und zwar wirkt Betahistin als inverser Agonist des H3-Rezeptors [23].

\section{Akute einseitige Vestibulopathie}

Symptome I Die Leitsymptome der akuten einseitigen Vestibulopathie (früher: „Neuritis vestibularis") sind

- akut einsetzender über Tage anhaltender Drehschwindel mit Scheinbewegungen („Oszillopsien“),

- Gangabweichung und Fallneigung (zur betroffenen Seite) sowie

- Übelkeit/Erbrechen ohne Hirnstamm- oder Kleinhirnsymptome und

- ohne akute Hörstörung.

Augenbewegungen und VOR I Bei der Untersuchung mittels M-Brille ( $\bullet$ Abb. 2 ) oder Frenzelbrille verhindern die vergrößernden Linsen zum einen die visuelle Fixationssuppression eines Spontannystagmus und erleichtern zum anderen die Beobachtung der Augenbewegungen des Patienten. Es findet sich ein peripherer vestibulärer Spontannystagmus zur nicht betroffenen Seite. Der Kopfimpulstest ist zur Seite der Läsion pathologisch. Es bestehen eine Fallneigung, Augenverrollung und Auslenkung der subjektiven visuellen Vertikalen zur betroffenen Seite.

Die virale Genese der akuten einseitigen Vestibulopathie ist wahrscheinlich, aber nicht bewiesen.

Therapie | Diese beruht auf drei Prinzipien:

1. symptomatische,

2. kausale und

3. physiotherapeutische Behandlung.

Symptomatische Therapie | Antivertiginosa (z.B. 100-300 mg Dimenhydrinat) sollten nur innerhalb der ersten Tage und nur bei schwerer Übelkeit und Brechreiz gegeben werden, da sie die zentrale Kompensation des peripheren Vestibularisausfalls verzögern.

„Kausale Therapie“ I Basierend auf der Annahme, dass die Neuritis vestibularis auf der Reaktivierung einer latenten Herpes simplex Virus 1 Infektion beruht, wurde eine vierarmige placebo-kontrollierte Studie durchgeführt. Diese zeigte, dass eine Monotherapie mit Glukokortikoiden (initial $100 \mathrm{mg} / \mathrm{d}$ Methylprednisolon, Dosis alle 3 Tage um $20 \mathrm{mg}$ reduzieren) zu einer signifikanten Verbesserung der Erholung der peripher vestibulären Funktion führt. Die Gabe von Valaciclovir bzw. die Kombination von Glukokortikoiden mit Valaciclovir hat keinen bzw. keinen zusätzlichen Effekt [24]. Die Wirkung von Cortison auf die Verbesserung der Funktion muss 
in weiteren kontrollierten multizentrischen Studien untersucht werden.

Physikalisch-medizinische Therapie | Die Förderung der zentralen Kompensation durch physikalische Therapie ist ein weiteres Behandlungsprinzip. Eine kontrollierte Studie zeigte, dass Gleichgewichtstraining die sog. vestibulo-spinale Kompensation und damit Haltungsregulation signifikant beschleunigt [25]. Zu den Übungen zählen willkürliche Augenbewegungen und Fixation zur Verbesserung der gestörten Blickstabilisation, aktive Kopfbewegungen zur Neueineichung des VOR sowie Balance-, Zielbewegungen und Gehübungen zur Verbesserung der vestibulo-spinalen Haltungsregulation und Zielmotorik $(3 \times 30$ Minuten pro Tag). Diese Befunde werden durch eine Cochrane-Analyse gestützt [26].

\section{Bilaterale Vestibulopathie}

Leitsymptome | Diese sind:

- bewegungsabhängiger Schwankschwindel mit Gang- und Standunsicherheit verstärkt in Dunkelheit und auf unebenem Grund (vestibulospinale Funktionsstörungen)

- in etwa $40 \%$ der Fälle „Wackeln der Umwelt“ (Oszillopsien) und unscharfes Sehen beim Gehen oder raschen Kopfbewegungen (Funktionsstörung des VOR)

Die betroffenen Patienten sind im Sitzen und Liegen typischerweise beschwerdefrei. Es kommt außerdem zu Störungen des räumlichen Gedächtnisses und der Navigation mit einer umschriebenen Hippocampusatrophie [27]. Die bilaterale Vestibulopathie ist die häufigste Ursache für bewegungsabhängigen Schwankschwindel beim älteren Patienten.

Ursache I Die Ursachen einer bilateralen Vestibulopathie sind vielfältig, bleiben aber in über 50\% der Fälle unklar. Die drei häufigsten nachweisbaren sind ototoxische Aminoglykoside, beidseitiger Morbus Menière und Meningitis [28]. Ein Zusammenhang mit degenerativen Kleinhirnerkrankungen ist inzwischen gut belegt [28-30]: sog. Cerebellar Ataxia with Neuropathy and Vestibular Areflexia Syndrome (CANVAS), bestehend aus bilaterale Vestibulopathie in Kombination mit sensorischer axonaler Polyneuropathie und cerebellärer Ataxie und Okulomotorikstörungen. Das Syndrom macht nach unserer Erfahrung etwa 30\% der bislang als idiopathisch eingeordneten Fälle aus.

Therapie | Da die häufigste nachweisbare Ursache einer bilateralen Vestibulopathie eine antibiotische Behandlung mit Aminoglykosiden ist [26], sollten diese sehr restriktiv eingesetzt werden, zumal der ototoxische Effekt erst mit Latenz von vielen Tagen einsetzt. Die physikalische Therapie mit Gang- und Gleichgewichtstraining erleichtert die Anpassung an den Funktionsausfall durch Förderung der visuellen und somatosensorischen Substitution. Zumindest für einseitige vestibuläre Funktionsstörungen konnte dies bestätigt werden [26]. Allein die Aufklärung über Ursache und Mechanismus führt häufig zu einer deutlichen Erleichterung der subjektiven Beschwerden. Die Diagnose einer bilateralen Vestibulopathie wird aber trotz vieler Arztbesuche meist noch zu spät gestellt, was die Beschwerden der Patienten weiter verstärkt.

\section{Vestibularisparoxysmie}

Kriterien | Die Diagnose der Vestibularisparoxysmie basiert auf folgenden Kriterien:

- mindestens 10 bis zu einer Minute dauernde spontan auftretende Schwindelattacken

- uniformer Verlauf beim individuellen Patienten

- Ansprechen auf eine Therapie mit Carbamazepin oder Oxcarbazepin [31,32]

Genese I Die Vestibularisparoxysmie entsteht wahrscheinlich durch hirnstammnahe neurovaskuläre Kompression des 8. Hirnnervs [31; 33] mit ephaptischen Fehlschlüssen teilweise demyelinisierter Axone. In über $95 \%$ lässt sich ein GefäßNerv-Kontakt im Austrittsbereich des VIII. Hirnnerven nachweisen, wobei es sich meist um die AICA (75\%), seltener um die A. vertebralis (10\%), eine Vene (10\%) oder die PICA (5\%) handelt [34]. Bei gesunden Kontrollen findet sich dieser aber auch in etwa $30-40 \%$, so dass die MRT-Untersuchung nicht spezifisch ist.

Therapie | Bei häufigen Attacken (>2 pro Monat) ist ein Therapieversuch mit Carbamazepin (200$600 \mathrm{mg} / \mathrm{d})$ oder Oxcarbazepin $(300-900 \mathrm{mg} / \mathrm{d})$ sinnvoll [35]. Bei Unverträglichkeit stehen alternativ Lamotrigin, Phenytoin oder Valproinsäure zur Verfügung. Bislang liegen aber für keine dieser Substanzen prospektive randomisierte kontrollierte Studien vor.

\section{Zentrale vestibuläre Syndrome}

Genese | Zentrale vestibuläre Syndrome entstehen überwiegend durch Läsionen der vestibulären Bahnen, die von den Vestibulariskernen im kaudalen Hirnstamm sowohl zum Cerebellum als auch zum Thalamus und vestibulären Kortex ziehen oder durch eine Schädigung des Vestibulocerebellums, selten durch „pathologische Erregung“ (paroxysmale Hirnstammattacken mit Ataxie bei MS; vestibuläre epileptische Aura).

Ursachen | Häufige Ursachen zentral vestibulären Schwindels sind die vestibuläre Migräne und ischämische Läsionen im Bereich des Hirnstamms. Ferner treten zentrale vestibuläre Störungen im Rahmen bestimmter Okulomotorikstörungen wie 
des Downbeat- und Upbeat-Nystagmus, in Form von Attacken bei der Episodischen Ataxie Typ 2 auf.

Vestibuläre Migräne | Nach den derzeitigen diagnostischen Kriterien ist die vestibuläre Migräne wie folgt definiert [36]:

- Sichere vestibuläre Migräne:

- A) mindestens 5 Episoden mit vestibulären Symptomen mittlerer oder starker Intensität und einer Dauer von $5 \mathrm{~min}-72 \mathrm{~h}$

- B) aktive oder frühere Migräne mit oder ohne Aura nach den Kriterien der ICHD

- C) ein/mehrere Migränesymptome während mindestens 50\% der vestibulären Episoden: Kopfschmerzen mit mindestens 2 der folgenden Merkmale (einseitige Lokalisation, pulsierender Charakter, mittlere oder starke Schmerzintensität, Verstärkung durch körperliche Routineaktivitäten), Photophobie und Phonophobie, visuelle Aura

D D) nicht auf eine andere vestibuläre oder ICHD-Diagnose zurückzuführen

- wahrscheinliche vestibuläre Migräne:

- A) mindestens 5 Episoden mit vestibulären Symptomen mittlerer oder starker Intensität und einer Dauer von $5 \mathrm{~min}-72 \mathrm{~h}$

- B) nur eines der beiden Kriterien B and C der vestibulären Migräne trifft zu (Migräneanamnese oder Migränesymptome während der Attacke)

- C) nicht auf eine andere vestibuläre oder ICHD-Diagnose zurückzuführen

Therapie I Bislang fehlen prospektive kontrollierte Therapiestudien. Deshalb kommt ein aktuelles Cochrane-Review zu dem Schluss, dass kontrollierte Studien zu dieser Erkrankung erforderlich sind [37].

Trotzdem werden bislang in Analogie zur Behandlung der Migräne die gleichen Prinzipien empfohlen, die sich in der Behandlung der Attacken und für die Migräneprophylaxe bewährt haben.

Mittel der 1. Wahl bei der Migräneprophylaxe sind in Abhängigkeit von den Kontraindikationen Betablocker (z.B. Metoprololsuccinat ca. $100 \mathrm{mg} / \mathrm{d}$ ), Topiramat oder Valproinsäure für die Dauer von 6 Monaten. Derzeit findet eine prospektive randomisierte placebo-kontrollierte multizentrische Studie zur Untersuchung des prophylaktischen Effekts von Metoprolol auf die Attackenfrequenz statt (PROVEMIG, BMBF gefördert).

\section{Downbeat- und Upbeat-Nystagmus}

Manifestation | Zwei zentrale Nystagmusformen mit jeweils vertikaler Schlagrichtung sind von besonderer Bedeutung: der Downbeat-Nystag- mus (DBN) (schnelle Phase schlägt nach unten) und der Upbeat -Nystagmus (UBN) (schnelle Phase schlägt nach oben). Der DBN ist der häufigste erworbene persistierende Nystagmus. Beide Formen manifestieren sich in erster Linie als Schwankschwindel und Gangunsicherheit und erst in zweiter Linie als Oszillopsien, d.h. Scheinbewegungen der Umwelt bedingt durch eine retinale Bildwanderung.

Fixationsnystagmus I Im Gegensatz zum o.g. Spontannystagmus handelt es sich bei beiden Formen um sog. Fixationsnystagmen, d.h. die Intensität nimmt bei Fixation nicht ab. DBN und UBN zeigen immer eine zentrale Störung an und haben besondere lokalisatorische Bedeutung. Dem DBN liegt meist eine beidseitige Flocculusfunktionsstörung zugrunde [38]. Die drei häufigsten Ursachen sind

- cerebelläre Atrophie,

- Ischämie und

- Arnold-Chiari-Malformation.

Der UBN (im Gegensatz zum DBN meist nur einige Wochen anhaltend) kann durch paramediane medulläre oder pontomesencephale Läsionen verursacht werden, wie z. B. bei Hirstamminfarkten oder-blutungen.

Therapie | Für den DBN liegen prospektive randomisierte, Placebo-kontrollierte Studien vor, die belegen, dass die Kaliumkanalblocker 3,4-Diaminopyridin [39] und 4-Aminopyridin (Dosierung $3 \times 5-10 \mathrm{mg}$ pro Tag) [40] zu einer signifikanten Abnahme des Nystagmus führen. Auch beim Upbeat-Nystagmus ist 4-Aminopyridin wirksam, wobei dazu bislang aber nur eine Einzelfallbeschreibung publiziert worden ist [41]. Die Behandlung wurde von den Patienten gut vertragen.

\section{Episodische Ataxie Typ 2 und andere Ataxien}

Auslöser und Ursache I Die familiären episodischen Ataxien sind seltene, autosomal dominante Erkrankungen mit zumindest zwei gut definierten Gruppen. Der Typ 2 (EA 2) ist durch rezidivierende, meist Stunden anhaltende Attacken mit Schwindel und Ataxie gekennzeichnet. Diese werden durch körperliche Aktivität, Stress oder Alkohol ausgelöst. Im Intervall finden sich bei über $90 \%$ der Patienten deutliche zentrale Okulomotorikstörungen, im Besonderen ein DBN. Ursache sind Mutationen im sog. CACNA1AGen bzw. im PQ-Calciumkanalgen.

Therapie | Auf der Basis einer prospektiven randomisierten kontrollierten Studie ist die Therapie der Wahl heute 4-Aminopyridin $(3 \times 5 \mathrm{mg}$ pro Tag) [42] bzw. Fampyra ( $2 \times 10 \mathrm{mg}$ pro Tag). Alternativ kann auch Acetazolamid (250-1000 mg pro Tag) gegeben werden. Aminopyridine sind eine 
Periphere vestibuläre Syndrome

Vestibularisparoxysmie

Neuritis vestibularis

BPPV
Carbamazepin vs Placebo

Betahistin (144 mg/d vs Placebo auf die zentr. Kompensation);

Vitamin D vs Placebo
VESPA

BETAVEST (Rekrutierung abgeschlossen)

VitD@BPPV

\section{Zentrale vestibuläre Syndrome}

\begin{tabular}{ll}
$\begin{array}{l}\text { episodische Ataxie } 2 \\
\text { vestibuläre Migräne }\end{array}$ & Fampyra vs Acetazolamid vs Placebo: \\
cerebelläre Ataxien & Beloc-Zok $(95 \mathrm{mg} / \mathrm{d}$ vs Placebo) \\
cerebelläre Gangstörungen & Acetyl-DL-Leucin vs Placebo \\
\hline
\end{tabular}

EAT-2-TREAT (Rekrutierung abgeschlossen) PROVEMIG

ALCAT (multinationale Studie)

FACEG (Rekrutierung abgeschlossen)

Tab. 4 Derzeit am Deutschen Schwindelzentrum durchgeführte prospektive placebo-kontrollierte Pharmakotherapiestudien bei verschiedenen Schwindelsyndromen. Es handelt sich jeweils um „investigator initiated trials“ (IITs). Die ersten 6 Studien werden vom Bundesministerium für Bildung und Forschung (BMBF) gefördert.

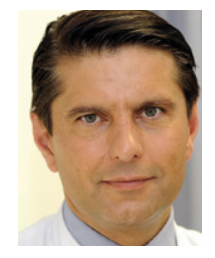

Prof. Dr. med. Michael Strupp

ist Oberarzt an der der Neurologischen Klinik des Klinikums der Universität München und am Deutschen Schwindel- und Gleichgewichtszentrum (DSGZ). Michael.Strupp@med. uni-muenchen.de

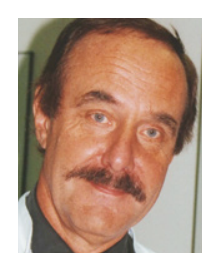

Prof. Dr. med. Thomas Brandt, FRCP

ist Direktor des DSGZ und Hertie Seniorprofessor des Zentrums für klinischen Neurowissenschaften am Klinikum der Universität München.

thomas.brandt@med. uni-muenchen.de

DOI $10.1055 / \mathrm{s}-0042-117336$ VNR2760512016149754016 Dtsch Med Wochenschr 2016; 141: 1698-1710 (c) Georg Thieme Verlag KG . Stuttgart . New York . ISSN 0012-0472 wirksame und in der eingesetzten niedrigen Dosierung gut verträgliche Behandlungsmöglichkeit für den DBN und UBN und die EA 2. Mit diesen Studien $[39,43,44]$ ließ sich auch ein neues Therapieprinzip erarbeiten: die Aktivierung von Purkinjezellen durch die Blockade von Kaliumkanälen verstärkt den inhibitorischen Einfluss von GABA auf vestibuläre und cerebelläre Kerngebiete und normalisiert die spontane Entladungsrate cerebellärer Purkinjezellen [45]. Aminopyridine sind auch bei der Behandlung cerebellärer Gangstörungen wirksam [46].

Perspektiven | Derzeit werden eine Reihe prospektiver randomisierter placebokontrollierter Therapiestudien, z. B. zum BPPV, zur Vestibularisparoysmie, der vestibulären Migräne und zur zentralen Kompensation bei akuter einseitigen Vestibulopathie/Neuritis vestibularis durchgeführt ( Tab. 4), die hoffentlich zu einer weiteren Verbesserung der Behandlung führen werden.

\section{Interessenkonflikt}

MS ist Joint Chief Editor beim Journal of Neurology, Editor in Chief bei Frontiers of Neuro-otology und Section Editor bei F1000. Er hat Honorare als Referent für Abbott, Actelion, Biogen, Eisai, GSK, Henning Pharma, Interacoustics, Merck, MSD, Otometrics, Pierre-Fabre, TEVA und UCB erhalten. Er ist als Consultant für Abbott, Actelion, Heel, IntraBio und Sensorion tätig. TB gibt an, dass kein Interessenkonflikt besteht.

\section{Konsequenz für Klinik und Praxis}

- Bei der Erhebung der Anamnese vier Aspekte herausarbeiten:

zeitlicher Verlauf

$\checkmark$ Art des Schwindels

> auslösende, verstärkende oder abschwächende Faktoren

- Begleitsymptome

- Bei der klinischen Untersuchung des vestibulären Systems auf einen Spontannystagmus achten, die Funktion des vestibulo-okulären-Reflexes testen und die Lagerungsmanöver sowie den Romberg durchführen.

- Klinische Zeichen zur Differenzierung zwischen akuten peripheren und zentralen vestibulären Syndromen sind vertikale Divergenz, zentraler Fixationsnystagmus, Blickrichtungsnystagmus, sakkadierte Blickfolge und normaler Kopfimpulstest.

- Therapie: Befreiungsmanöver sind beim BPPV sehr gut wirksam, hingegen ist das Evidenzniveau für die Pharmakotherapie vieler anderer Schwindelsyndrome noch niedrig, so dass weitere kontrollierte Studien notwendig sind.

\section{Literatur}

1 Corrales CE, Bhattacharyya N. Dizziness and death: An imbalance in mortality. Laryngoscope 2016; 126: 2134-2136

2 Neuhauser HK. Epidemiology of vertigo. Curr Opin Neurol 2007; 20: 40-46

3 Rieger A, Mansmann U, Maier W et al. [Management of patients with the cardinal symptom dizziness or vertigo]. Gesundheitswesen 2014; 76: e32-e38

Vollständiges Literaturverzeichnis unter http://dx.doi.org/10.1055/s-0042-117336 
4 Bremova T, Bayer O, Agrawal Y et al. Ocular VEMPs indicate repositioning of otoconia to the utricle after successful liberatory maneuvers in benign paroxysmal positioning vertigo. Acta Otolaryngol 2013; 133: 1297-1303

5 Feuerecker R, Habs M, Dieterich M, Strupp M. Chronic subjective dizziness: Fewer symptoms in the early morning--a comparison with bilateral vestibulopathy and downbeat nystagmus syndrome. J Vestib Res 2015; 25: 67-72

6 Halmagyi GM, Curthoys IS. A clinical sign of canal paresis. Arch Neurol 1988; 45: 737-739

7 Yip CW, Glaser M, Frenzel C et al. Comparison of the Bedside Head-Impulse Test with the Video Head-Impulse Test in a Clinical Practice Setting: A Prospective Study of 500 Outpatients. Front Neurol 2016; 7: 58

8 Strupp M, Fischer C, Hanss L, Bayer O. The takeaway Frenzel goggles: a Fresnel-based device. Neurology 2014; 83: 1241-1245

9 Zwergal A, Rettinger N, Frenzel C et al. A bucket of static vestibular function. Neurology 2009; 72 1689-1692

10 Dieterich M. Central vestibular disorders. J Neurol 2007; 254: 559-568

11 Cnyrim CD, Newman-Toker D, Karch C et al. Bedside differentiation of vestibular neuritis from central „vestibular pseudoneuritis“. J Neurol Neurosurg Psychiatry 2008; 79: 458-460

12 Lopez-Escamez JA, Carey J, Chung WH et al. Diagnostic criteria for Meniere's disease. J Vestib Res 2015; 25: 1-7

13 Strupp M, Kremmyda O, Adamczyk C et al. Central ocular motor disorders, including gaze palsy and nystagmus. J Neurol 2014; 261 Suppl 2: 542-558

14 Gurkov R, Flatz W, Louza J. In vivo visualization of endolyphatic hydrops in patients with Meniere's disease: correlation with audiovestibular function. Eur Arch Otorhinolaryngol 2011; 268: 1743-1748

15 Brandt T, Steddin S. Current view of the mechanism of benign paroxysmal positioning vertigo: cupulolithiasis or canalolithiasis? J Vestib Res 1993; 3: 373-382

16 Brandt T, Steddin S, Daroff RB. Therapy for benign paroxysmal positioning vertigo, revisited. Neurology 1994; 44: 796-800

17 Semont A, Freyss G, Vitte E. Curing the BPPV with a liberatory maneuver. Adv Otorhinolaryngol 1988; 42: 290-293

18 Brandt T, Dieterich M, Strupp M. Vertigo - Leitsymptom Schwindel. ed 2. Auflage, Heidelberg, Springer Medizin, 2012

19 Kim JS, Oh SY, Lee SH et al. Randomized clinical trial for apogeotropic horizontal canal benign paroxysmal positional vertigo. Neurology 2012; 78: 159-166

20 Adrion C, Fischer CS, Wagner J et al. Efficacy and safety of betahistine treatment in patients with Meniere's disease: primary results of a long term, multicentre, double blind, randomised, placebo controlled, dose defining trial (BEMED trial). BM] 2016; 352: h6816

21 Lezius F, Adrion C, Mansmann U et al. High-dosage betahistine dihydrochloride between 288 and $480 \mathrm{mg} /$ day in patients with severe Meniere's disease: a case series. Eur Arch Otorhinolaryngol 2011; 268: 1237-1240

22 Ihler F, Bertlich M, Sharaf K et al. Betahistine exerts a dose-dependent effect on cochlear stria vascularis blood flow in Guinea pigs in vivo. PLoS One 2012; 7: e39086

23 Bertlich M, Ihler F, Freytag S et al. Histaminergic $\mathrm{H}$-Heteroreceptors as a Potential Mediator of Betahistine-Induced Increase in Cochlear Blood Flow. Audiol Neurootol 2015; 20: 283-293
24 Strupp M, Zingler VC, Arbusow V et al. Methylprednisolone, valacyclovir, or the combination for vestibular neuritis. N Engl J Med 2004; 351: 354-361

25 Strupp M, Arbusow V, Maag KP et al. Vestibular exercises improve central vestibulospinal compensation after vestibular neuritis. Neurology 1998; 51: 838-844

26 McDonnell MN, Hillier SL. Vestibular rehabilitation for unilateral peripheral vestibular dysfunction. Cochrane Database Syst Rev 2015; 1: CD005397

27 Brandt T, Schautzer F, Hamilton D et al. Vestibular loss causes hippocampal atrophy and impaired spatial memory in humans. Brain 2005; 2732-2741

28 Zingler VC, Cnyrim C, Jahn K et al. Causative factors and epidemiology of bilateral vestibulopathy in 255 patients. Ann Neurol 2007; 61: 524-532

29 Kirchner H, Kremmyda O, Hufner K et al. Clinical, electrophysiological, and MRI findings in patients with cerebellar ataxia and a bilaterally pathological head-impulse test. Ann N Y Acad Sci 2011; 1233: 127-138

30 Wagner JN, Glaser M, Brandt T, Strupp M: Downbeat nystagmus: aetiology and comorbidity in 117 patients. J Neurol Neurosurg Psychiatry 2008; 79: 672-677

31 Brandt T, Dieterich M. Vestibular paroxysmia: vascular compression of the eighth nerve? Lancet 1994; 343: 798-799

32 Strupp M, Lopez-Escamez JA, Kim JS et al. Vestibular paroxysmia: diagnostic criteria. J Vestib Res. In press.

33 Strupp M, von Stuckrad-Barre S, Brandt T, Tonn JC. Teaching Neurolmages: Compression of the eighth cranial nerve causes vestibular paroxysmia. Neurology 2013; 80: e77

34 Best C, Gawehn J, Kramer HH et al. MRI and neurophysiology in vestibular paroxysmia: contradiction and correlation. J Neurol Neurosurg Psychiatry 2013; 84: 1349-1356

35 Strupp M, Dieterich M, Brandt T, Feil K. Therapy of Vestibular Paroxysmia, Superior Oblique Myokymia, and Ocular Neuromyotonia. Curr Treat Options Neurol 2016; 18: 34

36 Lempert T, Olesen J, Furman J et al. Vestibular migraine: diagnostic criteria. J Vestib Res 2012; 22: $167-172$

37 Maldonado FM, Birdi JS, Irving G] et al. Pharmacological agents for the prevention of vestibular migraine. Cochrane Database Syst Rev 2015; CD010600

38 Kalla R, Deutschlander A, Hufner K et al. Detection of floccular hypometabolism in downbeat nystagmus by fMRI. Neurology 2006; 66: 281-283

39 Strupp M, Schuler O, Krafczyk S et al. Treatment of downbeat nystagmus with 3, 4-diaminopyridine: a placebo-controlled study. Neurology 2003; 61: 165-170

40 Claassen J, Spiegel R, Kalla R et al: A randomised double-blind, cross-over trial of 4-aminopyridine for downbeat nystagmus--effects on slowphase eye velocity, postural stability, locomotion and symptoms. J Neurol Neurosurg Psychiatry 2013; 84: 1392-1399

41 Glasauer S, Kalla R, Buttner U. 4-aminopyridine restores visual ocular motor function in upbeat nystagmus. J Neurol Neurosurg Psychiatry 2005; 76: 451-453

42 Strupp M, Kalla R, Claassen J et al. A randomized trial of 4-aminopyridine in EA2 and related familial episodic ataxias. Neurology 2011; 77: 269-275

43 Strupp M, Kalla R, Dichgans M et al. Treatment of episodic ataxia type 2 with the potassium channel blocker 4-aminopyridine. Neurology 2004; 62: 1623-1625 
44 Kalla R, Glasauer S, Buttner U. 4-aminopyridine restores vertical and horizontal neural integrator function in downbeat nystagmus. Brain 2007; 130: 2441-2451

45 Alvina K, Khodakhah K: The therapeutic mode of action of 4-aminopyridine in cerebellar ataxia. J Neurosci 2010; 30: 7258-7268

46 Schniepp R, Wuehr M, Neuhaeusser $M$ et al.

4-aminopyridine and cerebellar gait: a retrospective case series. J Neurol 2012; 259: 2491-2493 


\section{CME-Fragen}

CME-Teilnahme

- Viel Erfolg bei Ihrer CME-Teilnahme unter http://cme.thieme.de

- Diese Fortbildungseinheit ist 12 Monate online für eine CME-Teilnahme verfügbar.

- Sollten Sie Fragen zur Online-Teilnahme haben, unter http:// cme.thieme.de/hilfe finden Sie eine ausführliche Anleitung.

1. Welches klinische Zeichen stützt die Diagnose einer akuten zentralen vestibulären Läsion?

a Skew deviation/vertikale Divergenz

b pathologischer Kopfimpulstest

c Nystagmus durch visuelle Suppression unterdrückbar

d vertikal torsioneller Lagerungsnystagmus

e pathologischer Rombergtest

2 Akut einsetzender über viele Tage anhaltender Drehschwindel ist typisch für

a Vestibularisparoxysmie

b akute einseitige Vestibulopathie

c benignen peripheren paroxysmalen Lagerungsschwindel

d bilaterale Vestibulopathie

e Downbeat-Nystagmussyndrom

3. Ein 34-jähriger Ingenieur berichtet über einen seit $\mathbf{5}$ Jahren bestehenden fluktuierenden Schwank- und Benommenheitsschwindel. Dieser tritt 30 min nach dem Aufwachen auf, verstärke sich in Menschenansammlungen und bessere sich nach leichtem Alkoholgenuss und bei sportlichen Aktivitäten. Keine Zunahme der Symptomatik im Dunkeln oder auf unebenem Untergrund. Was ist die wahrscheinliche Diagnose?

a Upbeat-Nystagmus

b bilaterale Vestibulopathie

c cervicogener/vertebragener Schwindel

d funktioneller/phobischer Schwankschwindel

e episodische Ataxie Typ 2

4. Welche Aussage zu Augenbewegungsstörungen und Nystagmus ist richtig?

a Ursache einer isolierten vertikalen Sakkadenparese ist Störung im Bereich des cerebellären Flocculus.

b Die internukleäre Ophthalmoplegie ist durch eine beidseitige horizontale Sakkadenparese gekennzeichnet.

c Der Downbeat-Nystagmus ist typisch für eine Funktionsstörung im frontalen Augenfeld.

d Hypermetrische Sakkaden zeigen eine Funktionsstörung im Mittelhirn an.

e Ein isolierter vertikaler Blickrichtungssnystagmus findet sich bei einer Funktionsstörung des Interstitiellen Nucleus Cajal.

5. Ein 42-jähriger Bauarbeiter erleidet ein leichtes Schädelhirntrauma. Am nächsten Tag bemerkt er beim Husten, Pressen auf der Toilette, Naseputzen sowie Heben schwerer Lasten kurzen Drehschwindel und hüpfende Bilder vor seinen Augen. Auch kann er seine eigene Stimme im linken Ohr deutlicher hören. Was ist die wahrscheinliche Diagnose?

a benigner peripherer paroxysmaler Lagerungsschwindel

b Morbus Menière

c Bogengangsdehiszenz/Perilymphfistel

d Vestibularisparoxysmie

e Traumatische Vertebralisdissektion

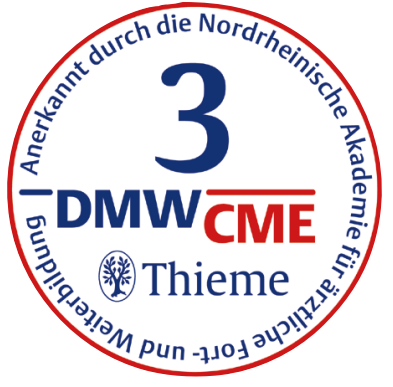

6. Welche Aussage zur klinischen Untersuchung ist richtig?

a Mit dem Kopfdrehtest wird die Funktion der Otolithen untersucht.

b Bei einer Canalolithiasis eines horizontalen Bogengangs findet sich ein linear-horizontaler Nystagmus.

c Mittels der Subjektiven Visuellen Vertikalen (SVV) lassen sich periphere von zentralen vestibulären Störungen differenzieren.

d Zur Diagnose eines Morbus Menière wird eine Hochtonschwerhörigkeit gefordert.

e Die Richtung eines Nystagmus wird entsprechend der langsamen Phase der Augenbewegungen angegeben.

7. Welches Medikament hat einen nachweisbaren Effekt bei der Behandlung des Morbus Menière?

a Aminopyridine

b Carbamazepin

c Gabapentin

d Gentamycin

e Metoprolol

8. Ein 75-jähriger Patient mit bekanntem Diabetes und arterieller Hypertonie leidet seit 3 Stunden unter einem erstmalig aufgetretenen heftigen Drehschwindel und einer deutlichen Fallneigung nach links sowie einer Sensibilitätsstörung in der linken Gesichtshälfte. Sie sehen einen horizontal-torsionellen Fixationsnystagmus nach rechts, der durch visuelle Fixation nicht unterdrückt werden kann. Der Kopfimpulstest ist regelrecht. Was ist die wahrscheinliche Diagnose?

a Morbus Menière

b vestibuläre Migräne

c Hirnstamminfarkt

d Lagerungsschwindel

e akute einseitige periphere Vestibulopathie

9. Ein 8-jähriges Mädchen berichtet über $2 \times$ pro Monat spontan auftretende Dreh- und Schwankschwindelattacken, die über 1-3 Stunden anhielten und mit Übelkeit, Licht- und Lärmempfindlichkeit und pulsierendem Kopfschmerz einhergingen. Keine Ohrsymptome. Die Untersuchung zwischen den Attacken ist regelrecht. In einer Attacke wurde ein Spontannystagmus beobachtet. Was ist die wahrscheinliche Diagnose? vestibuläre Migräne Vestibularisparoxysmie Morbus Menière funktioneller Schwindel orthostatische Dysregulation

10. Bei der akuten einseitigen peripheren Vestibulopathie findet sich bei klinischer Untersuchung

ein vertikaler Nystagmus

b ein allseitiger Blickrichtungsnystagmus

eine allseits sakkadierte Blickfolge

eine akute Hörminderung

ein pathologischer Kopfimpulstest 


\section{Angaben zur Person}

Name, Vorname, Titel:

Straße, Hausnr.:

PLZ, Ort:

Anschrift: $\square$ privat $\square$ dienstlich

Ich bin Mitglied der Ärztekammer (bitte Namen der Kammer eintragen):

Jahr meiner Approbation:

Ich befinde mich in der Weiterbildung zum:

Ich habe eine abgeschlossene Weiterbildung in ...

(bitte Fach eintragen):

Ich bin tätig als: $\square$ Assistenzarzt $\square$ Oberarzt $\square$ Chefarzt $\square$ niedergelassener Arzt $\square$ Sonstiges Ich bin DMW-Abonnent: $\square$ ja $\square$ nein

Falls nein: ich habe den Fragebogen aus / von:

$\square$ Thieme-connect $\square$ Kollegen $\square$ der Klinik $\square$ einer Bibliothek $\square$ Sonstiges

Lernerfolgskontrolle (Eine Antwort pro Frage ankreuzen)
1. $\square$ A $\square$ B $\square$ C $\square$ D $\square$ E
2. $\square$ A $\square$ B $\square \mathrm{C} \quad \square$ D $\square$ E
3. $\square$ A $\square$ B $\square$ C $\square$ D $\square$ E
4. $\square$ A $\square$ B $\square$ C $\square$ D $\square$ E
5. $\square$ A $\square$ B $\square$ C $\square$ D $\square$ E
6. $\square$ A $\square$ B $\square$ C $\square$ D $\square \mathrm{E}$
7. $\square$ A $\square$ B $\square$ C $\square$ D $\square$ E
8. $\square$ A $\square$ B $\square$ C $\square$ D $\square$ E
9. $\square \mathrm{A} \quad \square \mathrm{B} \quad \square \mathrm{C} \quad \square \mathrm{D} \quad \square \mathrm{E}$
10. $\square \mathrm{A} \quad \square$ B $\square \mathrm{C} \quad \square \mathrm{D} \quad \square \mathrm{E}$

Ich versichere, dass ich die Beantwortung der Fragen selbst und ohne fremde Hilfe durchgeführt habe

Ort, Datum:

Unterschrift:

Bitte in dieses Feld Ihre DMW Abonnement-Nummer eintragen:

\section{Fragen zur Zertifizierung}

1. Das Thema des Beitrages kommt in meiner ärztlichen Tätigkeit $\square$ häufig vor $\square$ selten vor $\square$ regelmäßig vor $\square$ gar nicht vor

2. Bei diesem Thema habe ich $\square$ eine feste Gesamtstrategie $\square$ keine Strategie $\square$ noch offene Einzelprobleme

3. In Bezug auf das Thema des Beitrages

$\square$ fühle ich mich nach dem Studium des Beitrags in meiner Strategie bestätigt

$\square$ habe ich meine Strategie verändert:

$\square$ habe ich erstmals eine einheitliche Strategie erarbeitet

$\square$ habe ich keine einheitliche Strategie ableiten können

4. Wurden aus der Sicht Ihrer täglichen Praxis heraus wichtige Aspekte des Themas

nicht erwähnt: $\square$ ja, welche $\square$ nein

zu knapp abgehandelt? $\square$ ja, welche $\square$ nein

überbewertet? $\square$ ja, welche $\square$ nein

5. Verständlichkeit des Beitrages

$\square$ Der Beitrag ist nur für Spezialisten verständlich

$\square$ Der Beitrag ist auch für Nicht-Spezialisten verständlich

6. Beantwortung der Fragen

$\square$ Die Fragen lassen sich aus dem Studium des Beitrags allein beantworten

$\square$ Die Fragen lassen sich nur unter Zuhilfenahme zusätzlicher Literatur beantworten

7. Die Aussagen des Beitrages benötigen eine ausführlichere Darstellung

$\square$ zusätzlicher Daten

$\square$ von Befunden bildgebender Verfahren

$\square$ die Darstellung ist ausreichend

8. Wieviel Zeit haben Sie für das Lesen des Beitrages und der Bearbeitung des Quiz benötigt?

Zertifizierungsfeld (wird durch die DMW ausgefüllt)

Ihr Ergebnis:

Sie haben von 10 Fragen richtig beantwortet.

Sie haben $\square$ bestanden und 3 Punkte erworben $\square$ nicht bestanden $\square$ ungültig, weil:

Stuttgart, den Stempel/Unterschrift 